\title{
Level of Consciousness
}

National Cancer Institute

\section{Source}

National Cancer Institute. Level of Consciousness. NCI Thesaurus. Code C88429.

The level of awareness of an organism. 